\title{
SUFFICIENT CONDITIONS FOR THE EXISTENCE OF LIMITING CARLEMAN WEIGHTS
}

\author{
PABLO ANGULO-ARDOY ${ }^{1}$, DANIEL FARACO ${ }^{2,3}$ and LUIS GUIJARRO ${ }^{2,3}$ \\ ${ }^{1}$ E.T.S de Ingenieros Navales, Universidad Politécnica de Madrid, Madrid, Spain; \\ email: pablo.angulo@uam.es \\ ${ }^{2}$ Department of Mathematics, Universidad Autónoma de Madrid, Madrid, Spain \\ ${ }^{3}$ ICMAT CSIC-UAM-UCM-UC3M, Madrid, Spain; \\ email: daniel.faraco@uam.es, luis.guijarro@uam.es
}

Received 8 April 2016; accepted 13 January 2017

\begin{abstract}
In Angulo-Ardoy et al. [Anal. PDE, 9(3) (2016), 575-596], we found some necessary conditions for a Riemannian manifold to admit a local limiting Carleman weight (LCW), based on the CottonYork tensor in dimension 3 and the Weyl tensor in dimension 4. In this paper, we find further necessary conditions for the existence of local LCWs that are often sufficient. For a manifold of dimension 3 or 4 , we classify the possible Cotton-York, or Weyl tensors, and provide a mechanism to find out whether the manifold admits local LCW for each type of tensor. In particular, we show that a product of two surfaces admits an LCW if and only if at least one of the two surfaces is of revolution. This provides an example of a manifold satisfying the eigenflag condition of AnguloArdoy et al. [Anal. PDE, 9(3) (2016), 575-596] but not admitting LCW.
\end{abstract}

2010 Mathematics Subject Classification: 35R30 (primary); 53A30 (secondary)

\section{Introduction}

Since the 1987 foundational paper of Sylvester and Uhlmann [18] (for more recent results see $[4,10,11])$, the only effective strategy to solve the Calderón inverse problem has been based on the existence of Complex Geometric Optic solutions. In the Riemannian setting, it was discovered in [8] that this type of solution depends on the existence of so called limiting Carleman weights. This notion was introduced by Kenig, Sjöstrand and Uhlmann in [13]. The existence of such functions was shown to be a problem in conformal geometry:

(c) The Author(s) 2017. This is an Open Access article, distributed under the terms of the Creative Commons Attribution licence (http://creativecommons.org/licenses/by/4.0/), which permits unrestricted re-use, distribution, and reproduction in any medium, provided the original work is properly cited. 
THEOREM 1 [8, Theorem 1.2]. If $(M, g)$ is an open manifold having a limiting Carleman weight, then some conformal multiple of the metric $g$, called $\tilde{g} \in[g]$, admits a parallel unit vector field. For simply connected manifolds, the converse is true.

For further developments, see [5, 7, 9, 12, 16, 17]. To avoid the simply connected hypothesis, we focus on existence of a local LCW at a point $p$, which is an LCW defined on some neighborhood of $p$.

Another way to reformulate Theorem 1 is by saying that locally, the metric $g$ is conformal to a product metric of the form $d t^{2}+g_{0}$ in $\mathbb{R} \times M_{0}$ for some $n-1$ dimensional manifold $M_{0}$; some of its limiting Carleman weights would be of the form $\phi(t, x)=a t+b$ for $a, b \in \mathbb{R}$.

Since this condition relates to the conformal class, the paper [2] studied such condition in terms of the classical tensors of conformal geometry, that is, the Weyl and Cotton tensors (see [2, Section 2] for the basics on algebraic curvature operators and bivectors over a real vector space $V$; we stick to the notation from that paper). In [2] we introduced the following notion:

Definition 2 [2]. Let $W$ be a Weyl tensor in $S^{2}\left(\Lambda^{2} V\right)$. We say that $W$ satisfies the eigenflag condition if and only if there is a nonzero vector $v \in V$ such that $W\left(v \wedge v^{\perp}\right) \subset v \wedge v^{\perp}$, where $v \wedge v^{\perp}$ denotes the set of bivectors

$$
\{v \wedge w: w \in V,\langle v, w\rangle=0\} .
$$

A 1-dimensional subspace of $V$ is called an eigenflag direction if it is spanned by some $v$ satisfying the above condition.

By examining the Weyl tensor of metrics with an $\mathbb{R}$-factor, we obtained in [2] the following obstruction for the existence of LCW's.

THEOREM 3 [2]. Let $(M, g)$ be a Riemannian manifold of dimension $n \geqslant 4$. Assume that a metric $\tilde{g} \in[g]$ admits a parallel vector field. Then for any $p \in M, W_{p}$ satisfies the eigenflag condition. In particular, for any $p \in M$, $W_{p} \in S^{2}\left(\Lambda^{2}\left(T_{p}^{*} M\right)\right)$ has at least $n-1$ linearly independent eigenvectors which are simple.

In dimension 3, the obstruction was described in terms of the Cotton-York tensor $C Y$.

THEOREM 4 [2]. Let $n=3$. If a metric $\tilde{g} \in[g]$ admits a parallel vector field, then for any $p \in M$, there is a tangent vector $v \in T_{p} M$ such that

$$
C Y_{p}(v, v)=C Y_{p}\left(w_{1}, w_{2}\right)=0
$$

for any pair of vectors $w_{1}, w_{2} \in v^{\perp}$. 
Both theorems only gave necessary conditions for the existence of LCW's. The results of the current paper study whether the converse to the above results hold. Specifically, we provide sufficient conditions for the existence of limiting Carleman weights, and thus Complex Geometric Optics solutions, and develop them in detail in dimensions 3 and 4.

For 3- and 4-dimensional manifolds, the results in this paper and in [2] can answer whether the manifold admits a limiting Carleman weight, and identify them if they exist, except for some corner cases that may require some ad hoc work. We show in Section 7 how to deal with these cases.

The proof of our results combines a more precise analysis of the algebraic structure of the Weyl and Cotton tensor and an analysis of how distributions in $T M$ get affected by conformal changes in the metric. With a slight abuse of notation we define

DEFINITION 5. $D \subset T M$ is a conformal factor of a metric $g$ if it is a smooth distribution of constant rank, such that a conformal multiple of $g$ is a product metric with $D$ and $D^{\perp}$ tangent to the factors.

When $\operatorname{dim}(D)=1$, Theorem 1 gives the connection between $D$ being a conformal factor, and the existence of LCW's. Nonetheless, even the case $1<\operatorname{dim}(D)<n-1$ is of independent interest, and can help to find LCW's. The reader can see this elsewhere in the paper, concretely in the proof of Theorem 11.

We prove that for a concrete distribution $D$, the behavior of the Lie derivatives or of the covariant derivatives characterizes conformal factors (see our Theorem 15). Thus, if for a metric $(M, g)$ there are a finite number of eigenflag conditions, the combination of this result for distributions of rank 1 and Theorems 3 and 4 answers the question of the existence of LCW.

In this way we can analyze all 3-dimensional manifolds.

THEOREM 6. Let $(M, g)$ be a 3-dimensional manifold, and $p \in M$ a given point.

(1) If $\operatorname{det} \mathrm{CY}_{p} \neq 0$, or there is a sequence of points $p_{k}$ converging to $p$ such that $\operatorname{det} \mathrm{CY}_{p_{k}} \neq 0$, there are no local LCW's at $p$.

(2) If there is a neighborhood $U$ of $p$ where $\mathrm{CY} \neq 0$ but $\operatorname{det} \mathrm{CY}=0$, then $U$ admits an $L C W$ if and only if one of the two 1-dimensional distributions of eigenflag directions for $\mathrm{CY}$ satisfies one of the equivalent conditions in Theorem 15.

(3) If there is a neighborhood $U$ of $p$ where $\left.\mathrm{CY}\right|_{U} \equiv 0$, then the metric is conformally flat in $U$, and it admits the same LCW's as a subset of $\mathbb{R}^{3}$. 
REMARK 7. Theorem 6 does not cover all the possibilities for 3-dimensional manifolds. There is one case missing, where $\mathrm{CY}_{p}=0$, but in any neighborhood of $p$ there are points with $\mathrm{CY}=0$, and points with $\operatorname{det} \mathrm{CY}=0$ but $\mathrm{CY} \neq 0$. In that case, the eigenflag direction fields obtained at points with $\operatorname{det} C Y=0$ but $\mathrm{CY} \neq 0$ approaching $p$ could loose smoothness (and even continuity!) at $p$, and no LCW could be obtained.

The analysis for the 4-dimensional case requires significantly more work. We start by dividing algebraic Weyl operators into types that depend on the different possibilities for their eigenflag directions.

LEMMA 8. The algebraic Weyl operators $W$ in a vector space of dimension 4 fall into one of the following types:

A W has no eigenflag directions.

B $W$ has at least one eigenflag direction and three different eigenspaces of dimension 2. In this case, $W$ has exactly four eigenflag directions.

C $W$ has at least one eigenflag direction and two eigenspaces with dimensions 4 and 2. In this case, the eigenflag directions for $W$ consist of the union of two orthogonal 2-planes.

D $W$ is null. All directions are eigenflag.

Once this is attained, we describe the existence of LCW's when the type of the Weyl curvature does not change in a neighborhood of a given point.

THEOREM 9. Let $(M, g)$ be a 4-dimensional manifold and $p \in M$.

- If $W$ is of type $A$ at $p$, or there is a sequence of points with Weyl tensors of type A converging to $p$, there are no local LCW's at $p$.

- If $W$ is of type $B$ at all points in a neighborhood of p, then $M$ admits a local $L C W$ at $p$ if and only if at least one of the four 1-dimensional distributions defined by eigenflags as in Lemma 8 satisfies the hypothesis of Theorem 15.

- If $W$ is of type $C$ at all points in a neighborhood of $p$, and the two complementary distributions of eigenflag directions satisfy the hypothesis of Theorem 15, then $M$ is locally conformal to a product of surfaces, and it admits a local LCW if and only if at least one of the two integral factors is a surface of revolution. 
- If $W$ is of type $D$ at all points in a neighborhood of $p$, then it is locally conformally flat at $p$, every direction is an eigenflag direction, and the metric admits the same LCW's as a subset of $\mathbb{R}^{4}$.

To write a concrete example of a manifold with an eigenflag vector field, but without any local LCW (showing that the necessary condition from Theorem 3 is not sufficient) recall that an ellipsoid in $\mathbb{R}^{3}$ is scalene if its three axis have different lengths.

THEOREM 10. Let $(M, g)=\left(S_{1}, g_{1}\right) \times\left(S_{2}, g_{2}\right)$ where $S_{1}$ and $S_{2}$ are two scalene ellipsoids with the metric induced by $\mathbb{R}^{3}$. Then any open subset of $(M, g)$ satisfies the eigenflag condition but does not admit any $L C W$.

It should be remarked that Liimatainen and Salo were the first to prove the existence of metrics for which there are no LCW's, and in fact gave a qualitative estimate of how large such set of metrics could be (see [15], and also [1]); later, [2] wrote the first explicit examples of such metrics.

The paper starts in Section 2 by considering a metric that becomes a product after a conformal change. The 3-dimensional case is examined in Section 3. Here Theorem 4 says that there are only a finite number of directions than can be factors of a product after conformal change; we analyze them by means of Theorem 15 . This will provide a proof of Theorem 6 .

Section 4 classifies the possible algebraic Weyl tensors that may arise in a 4-dimensional manifold in terms of its eigenflag directions and eigenspaces. This allows us to identify all the eigenflag directions.

We use this information in Section 5; as explained above, Theorem 15 suffices to deal with case B. In Section 6, we give an interesting example where three of the four eigenflag directions are conformal factors. This is a manifold with three LCW's with orthogonal level sets, that is not conformal to a product of surfaces.

We start Section 5 by proving that product metrics of surfaces have Weyl operators of Types $\mathrm{C}$ and $\mathrm{D}$; then we show that if such a product of surfaces admits an LCW and its Weyl operator is of type C (not the trivial conformally flat case), we can choose coordinates where the metric assumes a specially nice form. As a result, we are able to prove

THEOREM 11. Let $\left(S_{1}, g_{1}\right)$ and $\left(S_{2}, g_{2}\right)$ be open subsets of $\mathbb{R}^{2}$ with Riemannian metrics. Assume that the Weyl operator of the product metric does not vanish at any point. The following are equivalent:

- $\left(S_{1}, g_{1}\right)$ is locally isometric to a surface of revolution;

- $\left(S_{1}, g_{1}\right)$ has a nontrivial Killing vector field;

- $\left(S_{1} \times S_{2}, g_{1} \times g_{2}\right)$ admits an LCW that is everywhere tangent to the first factor. 
Hence Theorem 10 is a corollary of this, as scalene ellipsoids are not surfaces of revolution and their product satisfies the condition on the Weyl operator.

We are left with the case where a manifold may have a Weyl tensor of type C, but not be conformal to a product of surfaces. Therefore, in principle there are many candidates to be 1-dimensional conformal factors. In Section 7, we show that this is indeed possible, and show to deal with this situation in a specific example and explain how to proceed in general.

Finally, we would like to point out that, in principle, a similar analysis might be conducted in higher dimensions though satisfying the eigenflag condition is more rare (see [2, Theorem 6.1] for a quantitative statement in this regard), and the analysis is bound to become much more complicated.

\section{Criteria for a conformal product}

Suppose we are given a Riemannian manifold $(M, g)$ with a vector distribution $D \subset T M$ such that both $D$ and its orthogonal complement $D^{\perp}$ are integrable. The main result of this section establishes criteria that will assure that $M$ is locally conformal to a product metric with $D$ and $D^{\perp}$ tangent to the factors. In order to state them, we need some notation. We refer the reader to chapter 6 of [6] for a detailed introduction.

The orthogonal splitting $T M=D \oplus D^{\perp}$ induces bundle projections

$$
P_{D}: T M \rightarrow D, \quad P_{D^{\perp}}: T M \rightarrow D^{\perp} ;
$$

we respectively denote by $X^{D}$ and $X^{\perp}$ to the components of a vector field under the above splitting.

Given a metric tensor $g$ in $M$, we denote by $g^{D}$ and $g^{\perp}$ the restrictions of $g$ to $D$ and $D^{\perp}$, respectively, that is

$$
g^{D}(X, Y):=g\left(P_{D}(X), P_{D}(Y)\right), \quad g^{\perp}(X, Y):=g\left(P_{D^{\perp}}(X), P_{D^{\perp}}(Y)\right) .
$$

It is clear that

$$
g(X, Y)=g^{D}(X, Y)+g^{\perp}(X, Y),
$$

for any pair of vector fields $X, Y$.

Lemma 12. Assume $D, D^{\perp}$ are integrable distributions of dimensions $d$ and $n-d$, respectively. Then for any point $p \in M$, there is a coordinate chart $(U, \phi)$ with $\phi=\left(x_{1}, \ldots, x_{n}\right)$ such that:

(1) $\phi(p)=0, \phi(U)=(-1,1)^{n}$;

(2) the integral manifolds for $D$ in $U$ are given by equations

$$
\left(x^{d+1}, \ldots, x^{n}\right)=\left(a_{d+1}, \ldots, a_{n}\right) ;
$$


(3) the integral manifolds for $D^{\perp}$ in $U$ are given by equations

$$
\left(x^{1}, \ldots, x^{d}\right)=\left(a_{1}, \ldots, a_{d}\right) .
$$

Proof. Frobenius theorem gives us charts $\left(U_{1}, \phi_{1}\right)$ and $\left(U_{2}, \phi_{2}\right)$, with $\phi_{1}(p)=$ $\phi_{2}(p)=0, \phi_{1}=\left(y_{1}, \ldots, y_{n}\right), \phi_{2}=\left(z_{1}, \ldots, z_{n}\right)$, and such that the integral submanifolds for $D$ and $D^{\perp}$ are given respectively by the equations $\left(y_{d+1}, \ldots, y_{n}\right)$ $=\left(b_{d+1}, \ldots, b_{n}\right)$ and $\left(z_{1}, \ldots, z_{d}\right)=\left(c_{1}, \ldots, c_{d}\right)$ for constants $b_{i}, c_{j}$. The map $\phi=\left(z_{1}, \ldots, z_{d}, y_{d+1}, \ldots, y_{n}\right)$, defined in a neighborhood $U$ of $p$, is a local diffeomorphism at $p$ with $\phi(p)=0$, thus it defines a coordinate chart in some neighborhood of $p$. Taking a smaller neighborhood if needed, and with the help of a linear change of coordinates in $\mathbb{R}^{n}$, we can assume that its image is $(-1,1)^{n}$ as required.

It is clear from the Lemma that, if $N$ and $N^{\perp}$ are the integral manifolds for $D$ and $D^{\perp}$ through $p$ in $U$, then $U$ is diffeomorphic to $N \times N^{\perp}$. The aim of this section is to find conditions on a metric $g$ in $U$ such that $\left(\phi^{-1}\right)^{*} g$ is conformal to a product metric on $N \times N^{\perp}$. In order to do this, we need to introduce the following $1-$ form $\Phi$ in $M$ :

$$
\Phi(X):=\operatorname{Tr}_{g}\left(\frac{1}{d} \mathcal{L}_{X^{\perp}} g^{D}+\frac{1}{n-d} \mathcal{L}_{X^{D}} g^{\perp}\right) .
$$

Although the Lie derivative of some tensor $T, X \rightarrow \mathcal{L}_{X} T$, is not tensorial in $X$, we have that

$$
\begin{aligned}
\mathcal{L}_{f X^{\perp}} g^{D}(Y, Z) & =f \mathcal{L}_{X^{\perp}} g^{D}(Y, Z)+g^{D}\left(Y f X^{\perp}, Z\right)+g^{D}\left(Y, Z f X^{\perp}\right) \\
& =f \mathcal{L}_{X^{\perp}} g^{D}(Y, Z)
\end{aligned}
$$

and similarly for $\mathcal{L}_{X^{D}} g^{\perp}$, so $\Phi$ is actually a 1 -form.

DEFINITION 13. A distribution $D$ is said to be umbilical if there exists a vector field $H \in D^{\perp}$, called the mean curvature vector field of $D$, such that for $X, Y \in D$ and $Z \in D^{\perp}$ it holds that

$$
g\left(\nabla_{X} Y, Z\right)=g(X, Y) g(Z, H) .
$$

The relation of umbilicity to LCW's was already noted in [8].

REMARK 14. If $D$ is an arbitrary smooth distribution, then we can define the second fundamental form of the distribution

$$
I I(X, Y)=P_{D^{\perp}}\left(\nabla_{X} Y\right)
$$


where $P_{D^{\perp}}$ is the projection onto $D^{\perp}$ and $X, Y$ are vector fields tangent to $D$. It is easy to confirm that the distribution is integrable if and only if this form is symmetric, and in particular, an umbilical distribution is integrable.

THEOREM 15. Let $(M, g)$ be a Riemannian metric, and $D, D^{\perp}$ distributions as above.

The following are equivalent:

(1) $D$ and $D^{\perp}$ are integrable distributions and $g$ is locally conformal to the product of the metric restricted to an integral leave of $D$ and the metric restricted to an integral leave of $D^{\perp}$.

(2) The Lie derivative of $g^{D}$ with respect to any vector field in $D^{\perp}$ is a multiple of $g^{D}$, the Lie derivative of $g^{\perp}$ with respect to any vector field in $D$ is a multiple of $g^{\perp}$, and the 1-form $\Phi$ is closed.

(3) The distributions $D$ and $D^{\perp}$ are umbilic and, if $H_{1}$ and $H_{2}$ are the respective mean curvature vector fields, $\mathrm{H}_{1}+\mathrm{H}_{2}$ is a gradient vector field for the metric $g$.

Proof. (1) $\Longrightarrow$ (2) Let $\tilde{g}=e^{\alpha} g$ be a product metric of two metrics on the leaves of $D$ and $D^{\perp}$ through $p \in M$. Using the chart $(U, \phi)$, there are symmetric tensors in $U$ such that $\tilde{g}$ is written as

$$
\tilde{g}=\left(\begin{array}{ll}
e^{\alpha} g^{D} & \\
& e^{\alpha} g^{\perp}
\end{array}\right)
$$

where the first block does not depend on the coordinates of the second factor and vice versa. This is equivalent to

$$
\mathcal{L}_{X^{\perp}}\left(e^{\alpha} g^{D}\right)=\mathcal{L}_{X^{D}}\left(e^{\alpha} g^{\perp}\right)=0,
$$

and also to

$$
\begin{aligned}
& \mathcal{L}_{X^{\perp}}\left(g^{D}\right)=-X^{\perp}(\alpha) g^{D} \\
& \mathcal{L}_{X^{D}}\left(g^{\perp}\right)=-X^{D}(\alpha) g^{\perp} .
\end{aligned}
$$

Thus the first part of (2) is immediate, and the second follows because

$$
\Phi(X)=-X(\alpha)=-d \alpha(X) .
$$


(2) $\Longrightarrow$ (1) $D$ is integrable since, for $X, Y \in D$ and $Z \in D^{\perp}$ :

$$
\begin{aligned}
g^{\perp}([X, Y], Z) & =-\left(\mathcal{L}_{X} g^{\perp}\right)(Y, Z)+X\left(g^{\perp}(Y, Z)\right)-g^{\perp}(Y,[X, Z]) \\
& =-c g^{\perp}(Y, Z) \\
& =0
\end{aligned}
$$

where we are using that $\mathcal{L}_{X} g^{\perp}=c g^{\perp}$ for some function $c$. The integrability of $D^{\perp}$ is proved similarly.

Let $U$ be some simply connected neighborhood of a given point. Thus $\Phi$ being closed yields some function $\alpha$ such that $\Phi=-d \alpha$. For an arbitrary vector field $X$, the hypothesis in part (2) gives some function $c$ such that $\mathcal{L}_{X^{\perp}} g^{D}=c g^{D}$; taking traces in both sides, and using that the dimension of $D$ is $d$, we get that

$$
c=\frac{1}{d} \operatorname{Tr}_{g}\left(\mathcal{L}_{X^{\perp}} g^{D}\right)=\Phi\left(X^{\perp}\right)=-d \alpha\left(X^{\perp}\right)=-X^{\perp}(\alpha),
$$

thus $\mathcal{L}_{X^{\perp}} g^{D}+X^{\perp}(\alpha)=0$, and a simple computation yields $\mathcal{L}_{X^{\perp}}\left(e^{\alpha} g^{D}\right)=0$. A similar procedure gives us $\mathcal{L}_{X^{D}}\left(e^{\alpha} g^{\perp}\right)=0$, so the metric $\tilde{g}=e^{\alpha} g$ is a product of two metrics on the leaves of $D$ and $D^{\perp}$.

(1) $\Longrightarrow$ (3) Let $\tilde{g}=e^{2 \alpha} g$ be a product of two metrics on the leaves of $D$ and $D^{\perp}$ as before. A simple formula relates the Levi-Civita connections of $g$ and $\tilde{g}$ (see [3, Theorem 1.159]):

$$
\nabla_{X} Y=\tilde{\nabla}_{X} Y+g(X, Y) U-g(X, U) Y-g(Y, U) X
$$

where $U=-\nabla \alpha$. Thus for $X, Y \in D$ and $Z \in D^{\perp}$,

$$
g\left(\nabla_{X} Y, Z\right)=g(X, Y) g(U, Z)
$$

so the projection of $U$ onto $D^{\perp}$ is the mean curvature of $D$ and vice versa. It follows that $H_{1}+H_{2}=U$, which is a gradient.

(3) $\Longrightarrow$ (1) Suppose $H_{1}+H_{2}=-\nabla \alpha$, and define $\tilde{g}=e^{-2 \alpha} g$. Equation (4) shows that

$$
\begin{gathered}
g\left(\tilde{\nabla}_{X} Y, Z\right)=0 \\
g\left(\tilde{\nabla}_{Z} W, X\right)=0
\end{gathered}
$$

for $X, Y \in D$ and $Z, W \in D^{\perp}$.

As remarked before the Theorem, umbilic distributions are integrable, so by Lemma 12 , we can find a coordinate basis adapted simultaneously to $D$ and $D^{\perp}$, that is $\left\{\partial_{1}, \ldots, \partial_{d}\right\}$ span $D$ and $\left\{\partial_{d+1}, \ldots, \partial_{n}\right\}$ span $D^{\perp}$. Then for $i \in\{1 \ldots d\}$, $j, k \in\{d+1 \ldots n\}:$ 


$$
\begin{aligned}
\partial_{i} \tilde{g}_{j k} & =\partial_{i}\left(\tilde{g}\left(\partial_{j}, \partial_{k}\right)\right) \\
& =\tilde{g}\left(\tilde{\nabla}_{\partial_{i}} \partial_{j}, \partial_{k}\right)+\tilde{g}\left(\partial_{j}, \tilde{\nabla}_{\partial_{i}} \partial_{k}\right) \\
& =-\tilde{g}\left(\tilde{\nabla}_{\partial_{j}} \partial_{i}, \partial_{k}\right)-\tilde{g}\left(\partial_{j}, \tilde{\nabla}_{\partial_{k}} \partial_{i}\right)-\tilde{g}\left(\left[\partial_{i}, \partial_{j}\right], \partial_{k}\right)-\tilde{g}\left(\left[\partial_{i}, \partial_{k}\right], \partial_{j}\right) \\
& =-\partial_{j}\left(\tilde{g}\left(\partial_{i}, \partial_{k}\right)\right)+\tilde{g}\left(\partial_{i}, \tilde{\nabla}_{\partial_{j}} \partial_{k}\right)-\partial_{k}\left(\tilde{g}\left(\partial_{i}, \partial_{j}\right)\right)+\tilde{g}\left(\partial_{i}, \tilde{\nabla}_{\partial_{k}} \partial_{j}\right) \\
& =0
\end{aligned}
$$

where in the second line we have used the compatibility of the metric with the Levi-Civita connection, in the third we have used that the Levi-Civita connection is torsion-free, and in the fourth we have used Equations (5) and (6) together with the orthogonality of $D$ and $D^{\perp}$.

In a similar way, we also get the vanishing of $\partial_{i} \tilde{g}_{j k}$ when $j, k \in\{1 \ldots d\}$ and $i \in\{d+1 \ldots n\}$. Both sets of equalities imply that $\tilde{g}$ is a product metric.

Related conditions can be found in the literature. For example, [19] investigates the case of warped products. However, we have preferred to keep our criteria as simple as possible.

COROLlaRY 16. If $D$ is a 1-dimensional distribution with $D^{\perp}$ integrable, and $D$, $D^{\perp}$, satisfy any of the conditions (1)-(3) from Proposition 15 , then $(M, g)$ admits a limiting Carleman weight.

Proof. It follows directly from Theorem 1 and Proposition 15.

As mentioned in the Introduction, for a metric $g$ in the conditions of the above Corollary, $g$ would be locally isometric to a product $d t^{2}+g_{0}$ in $\mathbb{R} \times M_{0}$, and some of its limiting Carleman weights would be of the form $\phi(t, x)=a t+b$ for $a, b \in \mathbb{R}$.

\section{Conformal factors in dimension 3}

This section combines the results of the previous section with an examination of the Cotton-York tensor of a metric $g$ to describe when a 3-dimensional manifold has an LCW. We refer the reader to [2] for some background on the Cotton-York tensor. Recall that the space of algebraic Cotton-York tensors at some given point $p \in M$ coincides with the space of traceless symmetric operators in $T_{p} M$.

DEFINITION 17. An eigenflag direction of a traceless symmetric operator in a 3-dimensional euclidean space $V$ is a 1-dimensional vector subspace $L$ such that for any $v \in L$ and $w_{1}, w_{2} \in L^{\perp}$, we have

$$
C Y_{p}(v, v)=C Y_{p}\left(w_{1}, w_{2}\right)=0 .
$$


Suppose we are given a metric $g$ in $M$; [2, Theorem 1.6] shows that if a conformal metric $\tilde{g}$ admits a parallel vector field, the subspace $L$ that this vector field generates is an eigenflag direction of the Cotton-York tensor of $g$ at each point of $M$.

We start by classifying the possible algebraic Cotton-York tensors in terms of their eigenflag directions.

LEMMA 18. An algebraic Cotton-York tensor CY falls into one of the following categories:

- Every direction in $\mathrm{V}$ is eigenflag for $\mathrm{CY}$; this agrees with the case when $\mathrm{CY}$ is null.

- There are exactly two eigenflag directions for $\mathrm{CY}$; this agrees with the case when $\mathrm{CY}$ is not null and $\operatorname{det}(\mathrm{CY})=0$.

- There are no eigenflag directions; this is equivalent to the case $\operatorname{det}(\mathrm{CY}) \neq 0$.

Proof. Lemma 5.1 in [2] shows that $\operatorname{det}(\mathrm{CY})=0$ if and only if CY has an eigenflag direction, so assume that $L$ is an eigenflag direction for CY. If $v$ is a unit vector along $L$, and $\left\{v, w_{1}, w_{2}\right\}$ is an orthonormal basis of $V$, the matrix of CY is given by

$$
\left(\begin{array}{lll}
0 & a & b \\
a & 0 & 0 \\
b & 0 & 0
\end{array}\right)
$$

(see [2, Lemma 1.7]).

A further rotation with axis $L$ changes the matrix of CY to the form

$$
\left(\begin{array}{lll}
0 & c & 0 \\
c & 0 & 0 \\
0 & 0 & 0
\end{array}\right)
$$

If CY is not zero, the null directions for this symmetric operator is the union of the planes $\{x=0\}$ and $\{y=0\}$. Thus only the orthogonals to those two planes can be eigenflag directions.

Proof of Theorem 6. In the first case, we have a sequence of points $p_{k}$ converging to $p$ such that $\operatorname{det} \mathrm{CY}_{p_{k}} \neq 0$. By Lemma 18, there are no eigenflag directions at $p_{k}$, and thus there is not an open neighborhood of $p$ with a limiting Carleman weight.

Suppose next that there is a neighborhood $U$ of $p$ where $\operatorname{det} C Y \equiv 0$ but $\mathrm{CY} \neq 0$ anywhere. Lemma 18 says that there are two fields of eigenflag directions 
in $U$. Moreover, it is easy to see that such fields vary smoothly (see for instance, [14, Theorems 9.2.7 and 9.2.8]). Such fields are the only ones that can go in the direction of a conformal $\mathbb{R}$-factor; for this to happen, one of the two fields must satisfy the conditions of Proposition 15.

Finally, in the first case, the metric is conformally flat, since this is characterized by the vanishing of the Cotton-York tensor.

\section{Classification of algebraic Weyl tensors of 4-manifolds}

We would like to carry out a similar analysis for 4-dimensional manifolds to the one we did in Section 3; however, the extra dimension brings out a higher complexity, even at the level of algebraic Weyl operators. So in this section, we start by classifying such operators with respect to the size of their set of eigenflag directions.

In order to help the reader in the proof that follows, recall that, given a vector space, the Plücker relations are a set of algebraic relations that allow us to distinguish simple bivectors (that is, those of the form $v \wedge w$ for $v, w \in V$ ) from arbitrary bivectors (that is, elements in $\Lambda^{2}(V)$ ). In dimension 4, simple bivectors are characterized as those bivectors $\tau \in \Lambda^{2}(V)$ such that $\tau \wedge \tau=0$; choosing a base of $V,\left\{e_{i}\right\}$, the Plücker relations arise from writing $\tau$ in the base $\left\{e_{i} \wedge e_{j}\right\}$ and computing $\tau \wedge \tau$. This results in a single equation, of the form

$$
a_{12} a_{34}-a_{13} a_{24}+a_{14} a_{23}=0 \text { for } \tau=\sum_{1 \leqslant i<j \leqslant 4} a_{i j} e_{i} \wedge e_{j}
$$

Proof of Lemma 8. Let $W$ be a Weyl operator with an eigenflag direction $L=\langle v\rangle$. The operator $\left.W\right|_{\left\langle v \wedge v^{\perp}\right\rangle}$ is symmetric, and diagonalizes in an orthonormal basis $v \wedge e_{2}, v \wedge e_{3}, v \wedge e_{4}$. Define $e_{1}=v$ and $e_{i j}=e_{i} \wedge e_{j}$. It follows from the properties of the Weyl operator that $e_{23}, e_{24}$, and $e_{34}$ are eigenvectors of $W$ with the same eigenvalues as $e_{14}, e_{13}, e_{12}$, respectively (see the proof of [2, Theorem 6.1]). Thus $W$ diagonalizes as

$$
W=\left(\begin{array}{llllll}
\lambda_{12} & & & & & \\
& \lambda_{13} & & & & \\
& & \lambda_{14} & & & \\
& & \lambda_{12} & & \\
& & & \lambda_{13} & \\
& & & & \lambda_{14}
\end{array}\right) .
$$

Recall that $W$ is traceless, so $\lambda_{12}+\lambda_{13}+\lambda_{14}=0$. If the three numbers $\lambda_{12}, \lambda_{13}$, $\lambda_{14}$ are different, the operator has exactly three eigenspaces, each of dimension 2 . 
If two numbers coincide, there is one eigenspace of dimension 4 and a second eigenspace of dimension 2. Finally, the remaining possibility is that the Weyl operator vanishes. This will account for the different possibilities in the statement of the Theorem, once we have related them to the eigenflags. We do this case by case.

Three different eigenvalues. Suppose $\lambda_{12} \neq \lambda_{13} \neq \lambda_{14}$; then the eigenspace for $\lambda_{12}$ is the set of bivectors of the form $a e_{12}+b e_{34}, a, b \in \mathbb{R}$. The Plücker relations imply that such bivector is simple only when $a b=0$. In other words, the only simple bivectors in the eigenspace for $\lambda_{12}$ are the multiples of either $e_{12}$ or of $e_{34}$. Changing $i, j$ we get that every simple eigenvector of $W$ is a multiple of some $e_{i j}$, and consequently each one of the $e_{i}$ 's generates an eigenflag direction.

Suppose $v$ were a vector spanning a different eigenflag direction. Then $v \wedge v^{\perp}$ would be an eigenspace of $W$ consisting of simple bivectors, so there should be three orthogonal unit vectors $w_{k} \in v^{\perp}$ such that $v \wedge w_{k}$ are eigenvectors for $W$. It follows that $v \wedge w_{1}=e_{i j}$ for some $i, j$, which implies that $v, w_{1} \in \operatorname{span}\left(e_{i}, e_{j}\right)$, and we can assume without loss of generality that $i=1, j=2$. Then $w_{2} \in$ $\left(v, w_{1}\right)^{\perp}=\operatorname{span}\left(e_{3}, e_{4}\right)$; writing $v$ and $w_{2}$ in the basis $\left\{e_{i}\right\}$ and using that $v \wedge w_{2}$ can only be a coordinate 2-plane $\left\{e_{i} \wedge e_{j}\right.$, we obtain that $v=e_{1}$ or $v=e_{2}$.

Two different eigenvalues. Suppose $\lambda_{12}=\lambda_{13} \neq \lambda_{14}$. Let $v=a e_{2}+b e_{3}$ be any vector in $\operatorname{span}\left(e_{2}, e_{3}\right)$. Then

$$
v \wedge e_{1}=-a e_{12}-b e_{13}, \quad v \wedge e_{4}=a e_{24}+b e_{34},
$$

are eigenvectors of eigenvalue $\lambda_{12}$, while

$$
v \wedge\left(-b e_{2}+a e_{3}\right)=\left(a e_{2}+b e_{3}\right) \wedge\left(-b e_{2}+a e_{3}\right)=\left(a^{2}+b^{2}\right) e_{23}
$$

is an eigenbivector of eigenvalue $\lambda_{14}$. Therefore, $v \wedge v^{\perp}$ is an invariant subspace, and $v$ is an eigenflag direction. A similar argument applies to any vector in $\operatorname{span}\left(e_{1}, e_{4}\right)$ to show that it is an eigenflag direction, hence we only need to prove that there are no additional eigenflag directions.

A general bivector in the $\lambda_{12}$-eigenspace has the form $a e_{12}+b e_{34}+c e_{13}+d e_{24}$, and it is simple when

$$
a b-c d=0
$$

by the Plücker relations. This equation defines a 3-dimensional quadric in a 4dimensional space, and does not contain any linear space of dimension 3 . If $v$ is an eigenflag direction, $v \wedge v^{\perp}$ is an invariant subspace, and the restriction of $W$ to $v \wedge v^{\perp}$ diagonalizes in subspaces of the eigenspaces for $W$. Thus, $v \wedge v^{\perp}$ must intersect the eigenspace associated to $\lambda_{14}$. The intersection is spanned by a bivector $v \wedge w$, for some $w \in v^{\perp}$, but the only simple eigenbivectors in the eigenspace associated to $\lambda_{14}$ are $e_{14}$ and $e_{23}$. If $v \wedge w=e_{14}$, this implies that $v \in \operatorname{span}\left(e_{1}, e_{4}\right)$, while $v \wedge w=e_{23}$ implies $v \in \operatorname{span}\left(e_{2}, e_{3}\right)$. 


\section{Conformal factors in dimension 4}

A 4-dimensional manifold may be conformal to a product in two different ways: a product $\mathbb{R} \times N$, with $N$ a 3-dimensional manifold, or as a product of two surfaces. Since we are going to use the Weyl tensor to distinguish between the two cases, we start by computing the Weyl operator of a product of two surfaces.

LEMMA 19. Let $M_{1}$ and $M_{2}$ be two Riemannian surfaces.

(1) The Weyl operator of $M_{1} \times M_{2}$ has type $C$ or $D$ at any point.

(2) It has type $D$ at every point if and only if both surfaces have constant curvature $s_{1}$ and $s_{2}$, and $s_{1}+s_{2}=0$.

(3) If it has type $C$ at a point $p$, then $p$ has a neighborhood $U$ such $W_{q}$ has exactly two planes of eigenflag directions for every $q \in U$. These two distributions are the tangent distributions to the factors $M_{1}$ and $M_{2}$.

Proof. Taking isothermal coordinates $(t, x)$ on $M_{1}$ and $(y, z)$ on $M_{2}$, the product metric is written as

$$
g=\left(\begin{array}{llll}
f(t, x) & & & \\
& f(t, x) & & \\
& & h(y, z) & \\
& & & h(y, z)
\end{array}\right) .
$$

Denote the normalized coordinate fields as

$$
\hat{\partial}_{t}=\frac{1}{\sqrt{f}} \partial_{t}, \quad \hat{\partial}_{x}=\frac{1}{\sqrt{f}} \partial_{x}, \quad \hat{\partial}_{y}=\frac{1}{\sqrt{h}} \partial_{y}, \quad \hat{\partial}_{z}=\frac{1}{\sqrt{h}} \partial_{z} .
$$

In the basis of $\Lambda^{2} T_{p} M$

$$
\hat{\partial}_{t} \wedge \hat{\partial}_{x}, \quad \hat{\partial}_{t} \wedge \hat{\partial}_{y}, \quad \hat{\partial}_{t} \wedge \hat{\partial}_{z}, \quad \hat{\partial}_{y} \wedge \hat{\partial}_{z}, \quad \hat{\partial}_{x} \wedge \hat{\partial}_{z}, \quad \hat{\partial}_{x} \wedge \hat{\partial}_{y}
$$

the Weyl operator is diagonal; a long and tedious calculation by hand, that can be better carried out with some computer assistance, gives

$$
\begin{aligned}
\lambda \stackrel{\text { def }}{=} g\left(W\left(\hat{\partial}_{t} \wedge \hat{\partial}_{x}\right), \hat{\partial}_{t} \wedge \hat{\partial}_{x}\right)=g\left(W\left(\hat{\partial}_{y} \wedge \hat{\partial}_{z}\right), \hat{\partial}_{y} \wedge \hat{\partial}_{z}\right) \\
=\frac{h_{y}^{2}+h_{z}^{2}-h h_{y y}-h h_{z z}}{6 h^{3}}+\frac{f_{t}^{2}+f_{x}^{2}-f f_{t t}-f f_{x x}}{6 f^{3}}
\end{aligned}
$$


and

$$
\begin{aligned}
g\left(W\left(\hat{\partial}_{t} \wedge \hat{\partial}_{y}\right), \hat{\partial}_{t} \wedge \hat{\partial}_{y}\right) & =g\left(W\left(\hat{\partial}_{t} \wedge \hat{\partial}_{z}\right), \hat{\partial}_{t} \wedge \hat{\partial}_{z}\right) \\
& =g\left(W\left(\hat{\partial}_{x} \wedge \hat{\partial}_{y}\right), \hat{\partial}_{x} \wedge \hat{\partial}_{y}\right)=g\left(W\left(\hat{\partial}_{x} \wedge \hat{\partial}_{z}\right), \hat{\partial}_{x} \wedge \hat{\partial}_{z}\right) \\
& =-\frac{h_{y}^{2}+h_{z}^{2}-h h_{y y}-h h_{z z}}{12 h^{3}}-\frac{f_{t}^{2}+f_{x}^{2}-f f_{t t}-f f_{x x}}{12 f^{3}} \\
& =-\lambda / 2
\end{aligned}
$$

where $g$ above also denotes the metric induced on bivectors by the original metric tensor on the manifold. Thus $W$ falls into type $C$ or $D$ depending on whether $\lambda$ is different or equal to zero.

We recognize

$$
\lambda(t, x, y, z)=\frac{-1}{3}\left(s_{1}(t, x)+s_{2}(y, z)\right),
$$

where $s_{i}$ is the Gaussian curvature of $M_{i}$. Thus if $\lambda$ vanishes identically, $s_{1}$ and $s_{2}$ are global constants whose sum is 0 .

If $\lambda$ does not vanish at point $p=\left(p_{1}, p_{2}\right)$, let $U \subset M_{1} \times M_{2}$ be a neighborhood of $p$ where $\lambda$ does not vanish. Then Lemma 8 proves that at every $q \in U$, there are exactly two planes of eigenflag directions of $W_{p}$. It is simple, using (8) and (9), to prove directly that any vector in $\left\langle\partial_{t}, \partial_{x}\right\rangle$ spans an eigenflag direction.

Hence the eigenflag directions are exactly the tangent lines to either factor.

We are now ready to distinguish those metric products of surfaces that admit, simultaneously, an LCW.

LEMMA 20. Let $(M, g)$ be a product of surfaces that admits a limiting Carleman weight $\varphi$. Let $p \in M$ with $W_{p} \neq 0$; then there are coordinates $(t, x, y, z)$ around $p$ in which $g$ is written as

$$
g=\left(\begin{array}{llll}
e^{J(x)} & & & \\
& e^{J(x)} & & \\
& & e^{K(y, z)} & \\
& & & e^{K(y, z)}
\end{array}\right)
$$

for some functions $J=J(x), K=K(y, z)$.

Proof. Since $W_{p} \neq 0$, Lemma 19 implies that $W$ is of type $C$ in some neighborhood of $p$, and the set of eigenflag directions for the Weyl tensor of $g$ and $\tilde{g}$ is the union of the two planes $\left\langle\partial_{t}, \partial_{x}\right\rangle$ and $\left\langle\partial_{y}, \partial_{z}\right\rangle$. 
Choose some coordinate system $(t, x, y, z)$ adapted to the product structure $M_{1} \times M_{2}$, as in the proof of Lemma 19 .

Let $\tilde{g}$ be the rescaled metric

$$
\tilde{g}=|\nabla \varphi|^{2} g .
$$

Lemmas 3.10 and 3.11 in [16] give that the vector field $A=\tilde{\nabla} \varphi$ is a parallel vector field for $\tilde{g}$. Theorem 1.3 in [2] shows that $\langle A\rangle$ must be an eigenflag direction.

Without loss of generality, we assume that $A$ is in $\left\langle\partial_{t}, \partial_{x}\right\rangle$ at every point, hence there are functions $\alpha, \beta$ such that

$$
A=\alpha \partial_{t}+\beta \partial_{x}
$$

It follows from the definition of parallel vector field that $\Delta$, the distribution orthogonal to $A$, is integrable (see the proof of [16, Lemma 3.12, page 57]). It is clear that

$$
\Delta=\left\langle-\beta \partial_{t}+\alpha \partial_{x}, \partial_{y}, \partial_{z}\right\rangle .
$$

Denote $B:=-\beta \partial_{t}+\alpha \partial_{x}$; the integrability condition implies that

$$
\left[B, \partial_{y}\right]=\left(\partial_{y} \beta\right) \partial_{t}-\left(\partial_{y} \alpha\right) \partial_{x} \in \Delta
$$

and therefore $\left[B, \partial_{y}\right]$ must be in the $B$ direction, that is, $\left[B, \partial_{y}\right]=\lambda_{1} B$ for some function $\lambda_{1}$; thus

$$
\partial_{y} \beta=-\lambda_{1} \cdot \beta, \quad \partial_{y} \alpha=-\lambda_{1} \cdot \alpha .
$$

Looking at $\left[B, \partial_{z}\right]$, we get

$$
\partial_{z} \beta=-\lambda_{2} \cdot \beta, \quad \partial_{z} \alpha=-\lambda_{2} \cdot \alpha .
$$

Next, observe that any integral submanifold of $\Delta$ is foliated by surfaces tangent to $\left\langle\partial_{y}, \partial_{z}\right\rangle$; since $A$ is a parallel vector field, its integral curves are given by geodesics. Therefore, if we consider the maps

$$
\phi_{\left(t_{0}, x_{0}\right)}(u, v, w)=\exp _{\left(t_{0}, x_{0}, v, w\right)}(u A),
$$

we see that for each $\left(t_{0}, x_{0}\right)$, the vector fields $\partial_{u}, \partial_{v}, \partial_{w}$ are mapped by the differential of $\phi_{\left(t_{0}, x_{0}\right)}$ to $A, \partial_{y}$ and $\partial_{z}$, and therefore

$$
\left[A, \partial_{y}\right]=\left[A, \partial_{z}\right]=0 .
$$

On the other hand, writing $A=\alpha \partial_{t}+\beta \partial_{x}$, we would get

$$
\left[A, \partial_{y}\right]=-\left(\partial_{y} \alpha\right) \partial_{t}-\left(\partial_{y} \beta\right) \partial_{x}, \quad\left[A, \partial_{z}\right]=-\left(\partial_{z} \alpha\right) \partial_{t}-\left(\partial_{z} \beta\right) \partial_{x} .
$$


The consequence of this is that $\lambda_{1}=\lambda_{2}=0$, and every Lie bracket between elements in the basis $\left\{A, B, \partial_{y}, \partial_{z}\right\}$ vanishes, and hence they form a coordinate basis for some set of coordinates about $p$. We keep on denoting them $(t, x, y, z)$, although only the last two would coincide with the former. The first two, $(t, x)$, will still, however, parametrize the first factor of the product structure of $M$. In this chart, $\tilde{g}$ is written as

$$
\tilde{g}=\left(\begin{array}{llll}
1 & & & \\
& 1 & & \\
& & b(t, x, y, z) & \\
& & & b(t, x, y, z)
\end{array}\right)
$$

where $b$ is actually $h(y, z)|\nabla \varphi|^{2}(t, x, y, z)$.

Because $A$ is parallel, it follows from Koszul formula

$$
\begin{aligned}
2 g\left(\nabla_{X} Y, Z\right)= & \partial_{X}(g(Y, Z))+\partial_{Y}(g(X, Z))-\partial_{Z}(g(X, Y)) \\
& +g([X, Y], Z)-g([X, Z], Y)-g([Y, Z], X),
\end{aligned}
$$

that $b$ does not depend on $t$ (taking $X=A, Y=Z=\partial_{y}$ ).

The metric $\tilde{g}$ is conformal to a product where the factors are tangent to $\left\langle\partial_{t}, \partial_{x}\right\rangle=\langle A, B\rangle$ and $\left\langle\partial_{y}, \partial_{z}\right\rangle$. Let $\hat{\partial}_{y}=(1 / \sqrt{b}) \partial_{y}$ be a unit vector in the direction of $\partial_{y}$. We compute the Weyl operator in the basis

$$
A \wedge B, \quad A \wedge \hat{\partial}_{y}, \quad A \wedge \hat{\partial}_{z}, \quad \hat{\partial}_{y} \wedge \hat{\partial}_{z}, \quad B \wedge \hat{\partial}_{z}, \quad B \wedge \hat{\partial}_{y} .
$$

The $6 \times 6$ matrix of $W$ in this basis has the block structure

$$
W=\left(\begin{array}{cc}
W_{1} & 0 \\
0 & W_{1}
\end{array}\right)
$$

where the $3 \times 3$ block $W_{1}$ is

$$
\left(\begin{array}{ccc}
-\Psi & \frac{b b_{x y}-b_{x} b_{y}}{4 b^{5 / 2}} & \frac{b b_{x z}-b_{x} b_{z}}{4 b^{5 / 2}} \\
\frac{b b_{x y}-b_{x} b_{y}}{4 b^{5 / 2}} & \Psi / 2 & 0 \\
\frac{b b_{x z}-b_{x} b_{z}}{4 b^{5 / 2}} & 0 & \Psi / 2
\end{array}\right)
$$

where

$$
\Psi=\frac{b b_{x}^{2}-b^{2} b_{x x}-b_{y}^{2}+b b_{y y}-b_{z}^{2}+b b_{z z}}{6 b^{3}} .
$$


As mentioned in the proof of Lemma 19, if the metric is a product of surfaces tangent to $\left\langle\partial_{1}, \partial_{2}\right\rangle$ and $\left\langle\partial_{3}, \partial_{4}\right\rangle$, the off-diagonal elements in the corresponding basis of $\Lambda^{2}\left(T_{p} M\right)$ must vanish at every $p$. Thus:

$$
\begin{aligned}
& b b_{x y}=b_{x} b_{y} \\
& b b_{x z}=b_{x} b_{z} .
\end{aligned}
$$

Integrating the first equation we get:

$$
\begin{aligned}
\frac{\left(b_{x}\right)_{y}}{b_{x}} & =\frac{b_{y}}{b} \\
\log \left(b_{x}\right) & =\log (b)+C(x, z) \\
\frac{b_{x}}{b} & =e^{C(x, z)} \\
\log (b) & =D(x, z)+E(y, z)
\end{aligned}
$$

and similarly, integrating the second, we get $\log (b)=F(x, y)+G(y, z)$. Define $r=\log (b)$, and observe that $r_{x y}=r_{x z}=0$, which in turn yields $r_{x}=H(x)$, or $r(x, y, z)=-J(x)+K(y, z)$.

Thus, in the basis $\left\{A, B, \partial_{y}, \partial_{z}\right\}$, the metric $\tilde{g}$ is written as

$$
\tilde{g}=\left(\begin{array}{llll}
1 & & & \\
& 1 & & \\
& & e^{-J(x)} e^{K(y, z)} & \\
& & & e^{-J(x)} e^{K(y, z)}
\end{array}\right) .
$$

Undoing the change from $g$ to $\tilde{g}$ shows that the matrix for $g$ is as claimed in the statement of the Theorem.

REMARK 21. For a metric of the form (10), the vector field $\partial_{t}$ is a parallel unit vector field for the metric $\tilde{g}=e^{-J(x)} g$, and it is the gradient of the coordinate function $(t, x, y, z) \rightarrow t$. By [8, Lemma 2.5], the function $t$ is an LCW for $\tilde{g}$, and by $[8$, Lemma 2.1], it is also an LCW for $g$ (since the identity $(\tilde{M}, \tilde{g}) \rightarrow(M, g)$ is a conformal transformation).

We can now proceed with the proof of Theorem 11.

Proof of Theorem 11. Assume $S_{1} \times S_{2}$ has an LCW tangent to the first factor; Lemma 20 shows that there are coordinates in $S_{1}$ such that $g_{1}$ can be written in the form:

$$
g=\left(\begin{array}{cc}
e^{J(x)} & \\
& e^{J(x)}
\end{array}\right) .
$$

Clearly such a metric has $\partial_{t}$ as a Killing field. 
Assume now $\left(S_{1}, g_{1}\right)$ has a Killing field. Since for any vector field $X$ that does not vanish at a point $p$, there is a coordinate chart around a $p$ such that $X=\partial_{1}$, we can assume, using that $X$ is Killing, that there are coordinates where $g_{1}$ is written as before. It is then clear that after a change of coordinates of the form $(t, x) \rightarrow(t, \lambda(x))$, with $\lambda^{\prime}(x)=e^{J(x) / 2}$, the matrix of $g$ becomes

$$
g=\left(\begin{array}{ll}
e^{-J(x)} & \\
& 1
\end{array}\right)
$$

which is a piece of a surface of revolution.

Finally, if $\left(S_{1}, g_{1}\right)$ is a surface of revolution, take a chart as above; undoing the change of coordinates $(t, x) \rightarrow(t, \lambda(x))$ get a chart in the product where the metric $g_{1} \times g_{2}$ takes the expression appearing in Lemma 20. Multiplying by the conformal factor $e^{-J(x)}$ shows that there is an LCW along $\partial_{t}$.

Proof of Theorem 9. Theorem 1.3 in [2] shows that if $W_{p}$ has type A, there cannot be LCW's around $p$. Clearly, the same thing happens if $p$ is in the closure of the set of points with Type A Weyl operators.

If $p$ has a neighborhood where every point has a Type $\mathrm{C}$ Weyl operator, Lemma 8 shows that there are two orthogonal distributions $D$ and $D^{\perp}$; the hypothesis of the Theorem assure that there is a conformal change such that a neighborhood of $p$ is isometric to a product of surfaces; since one of them has a Killing field, there is an LCW.

The case of a Type B neighborhood is similarly done. Finally, if $W$ vanishes in a neighborhood of $p$, the manifold would be conformally flat around $p$.

\section{A metric with Weyl tensor of constant type $B$, three of the four eigenflag directions are conformal factors}

Lemma 8 has an interesting consequence: if a manifold admits an LCW, then it has at least four vector fields of eigenflag directions. In general, only one of them will really correspond to a conformal factor. Theorem 22 below shows a less usual case of a manifold with Weyl tensor of constant type B that is conformal to a product along three of the four eigenflag directions.

THEOREM 22. Take coordinates $(t, x, y, z)$ on an open set

$$
U \subset\left\{(t, x, y, z) \in \mathbb{R}^{4}: x>0\right\}
$$


Define the metric

$$
g=\left(\begin{array}{cccc}
1 & 0 & 0 & 0 \\
0 & 1 & 0 & 0 \\
0 & 0 & x & 0 \\
0 & 0 & 0 & x^{2}
\end{array}\right)
$$

on U. Then:

- The Weyl tensor of $g$ has type $B$ at every point.

- The eigenflag directions of the Weyl tensor are spanned by the coordinate vector fields.

- The functions $t, y, z$ are LCW's.

- The function $x$ is not an $L C W$.

Proof. The nonzero Christoffel symbols for this metric are

$$
\Gamma_{y y}^{x}=-1 / 2, \quad \Gamma_{z z}^{x}=-x, \quad \Gamma_{x y}^{y}=\Gamma_{y x}^{y}=\frac{1}{2 x}, \quad \Gamma_{x z}^{z}=\Gamma_{z x}^{z}=\frac{1}{x},
$$

the $(4,0)$ Riemann curvature tensor is

$$
\operatorname{Riem}(g)=\frac{1}{x}(\mathrm{~d} x \wedge \mathrm{d} y) \otimes(\mathrm{d} x \wedge \mathrm{d} y)+2 x(\mathrm{~d} y \wedge \mathrm{d} z) \otimes(\mathrm{d} y \wedge \mathrm{d} z),
$$

the Ricci tensor is

$$
\operatorname{Ric}(g)=\frac{1}{4 x^{2}} \mathrm{~d} x \otimes \mathrm{d} x-\frac{1}{4 x} \mathrm{~d} y \otimes \mathrm{d} y-\frac{1}{2} \mathrm{~d} z \otimes \mathrm{d} z,
$$

and the scalar curvature is $-1 / 2 x^{2}$.

We define the normalized vector fields $\hat{\partial}_{y}=\left(\partial_{y} / \sqrt{x}\right)$ and $\hat{\partial}_{z}=\left(\partial_{z} / x\right)$.

In the orthonormal basis of $\Lambda^{2} T_{p} U$

$$
\partial_{t} \wedge \partial_{x}, \quad \partial_{t} \wedge \hat{\partial}_{y}, \quad \partial_{t} \wedge \hat{\partial}_{z}, \quad \hat{\partial}_{y} \wedge \hat{\partial}_{z}, \quad \partial_{x} \wedge \hat{\partial}_{z}, \quad \partial_{x} \wedge \hat{\partial}_{y}
$$

the Weyl tensor of $g$ is diagonal, with a $3 \times 3$ block repeated twice

$$
W=\left(\begin{array}{ll}
W_{1} & \\
& W_{1}
\end{array}\right), \quad W_{1}=\left(\begin{array}{ccc}
-\frac{5}{96 x^{2}} & 0 & 0 \\
0 & \frac{1}{96 x^{2}} & 0 \\
0 & 0 & \frac{1}{24 x^{2}}
\end{array}\right) .
$$


The proof of Lemma 8 shows that the four eigenflag directions are spanned by $\partial_{t}$, $\partial_{x}, \partial_{y}$, and $\partial_{z}$.

It is obvious that $\partial_{t}$ is a unit parallel vector field, while $\partial_{y}$ and $\partial_{z}$ are unit parallel vector fields for the conformal metrics $1 / x g$ and $1 / x^{2} g$, respectively. Thus, according to Theorem 1, the coordinate functions $t, y$, and $z$ are LCW's.

In order to check if $\left\langle\partial_{x}\right\rangle$ is a conformal factor, we need to apply our Theorem 15. The second fundamental form of $\left\{\partial_{x}\right\}^{\perp}$ in the orthonormal basis $\left\{\partial_{t}, \hat{\partial}_{y}, \hat{\partial}_{z}\right\}$ is:

$$
\left(\begin{array}{ccc}
0 & 0 & 0 \\
0 & -\frac{1}{2 x} & 0 \\
0 & 0 & -\frac{1}{x}
\end{array}\right)
$$

which is not a multiple of the identity. This means that $\left\{\partial_{x}\right\}^{\perp}$ is not umbilical, and we deduce that $\left\langle\partial_{x}\right\rangle$ is not a conformal factor.

\section{A metric not conformal to a product, but with Weyl tensor of constant type $\mathbf{C}$}

In this example we show an explicit metric whose Weyl tensor has type $\mathrm{C}$ at all points in an open set, but which is not conformal to a product. After that, we show how to find out all its LCW's.

THEOREM 23. Let $U$ be any open subset of $\left\{(t, x, y, z) \in \mathbb{R}^{4}: x>0\right\}$. The metric

$$
g=\left(\begin{array}{cccc}
1 & 0 & 0 & 0 \\
0 & 1 & 0 & 0 \\
0 & 0 & x^{3} & 0 \\
0 & 0 & 0 & 1 / x
\end{array}\right)
$$

on $U$ has Weyl tensor of type $C$ at every point, but is not conformal to a product of surfaces. Moreover, there are only three LCW's which are the coordinate functions $t, y, z$.

Proof. The nonzero Christoffel symbols for this metric are

$$
\Gamma_{y y}^{x}=-\frac{3}{2} x^{2}, \quad \Gamma_{z z}^{x}=\frac{1}{2 x^{2}}, \quad \Gamma_{x y}^{y}=\Gamma_{y x}^{y}=\frac{3}{2 x}, \quad \Gamma_{x z}^{z}=\Gamma_{z x}^{z}=-\frac{1}{2 x},
$$

the $(4,0)$-Riemann curvature tensor is 


$$
\begin{aligned}
\operatorname{Riem}(g)= & -3 x(\mathrm{~d} x \wedge \mathrm{d} y) \otimes(\mathrm{d} x \wedge \mathrm{d} y) \\
& -\frac{3}{x^{3}}(\mathrm{~d} x \wedge \mathrm{d} z) \otimes(\mathrm{d} x \wedge \mathrm{d} z)-3(\mathrm{~d} y \wedge \mathrm{d} z) \otimes(\mathrm{d} y \wedge \mathrm{d} z),
\end{aligned}
$$

the Ricci tensor is

$$
\operatorname{Ric}(g)=-\frac{3}{2 x^{2}} \mathrm{~d} x \otimes \mathrm{d} x,
$$

and the scalar curvature is $-3 / 2 x^{2}$.

We define the normalized vector fields $\hat{\partial}_{y}=x^{-3 / 2} \partial_{y}$ and $\hat{\partial}_{z}=\sqrt{x} \partial_{z}$.

In the orthonormal basis of $\Lambda^{2} T_{p} U$

$$
\partial_{t} \wedge \partial_{x}, \quad \partial_{t} \wedge \hat{\partial}_{y}, \quad \partial_{t} \wedge \hat{\partial}_{z}, \quad \hat{\partial}_{y} \wedge \hat{\partial}_{z}, \quad \partial_{x} \wedge \hat{\partial}_{z}, \quad \partial_{x} \wedge \hat{\partial}_{y}
$$

the Weyl tensor of $g$ is diagonal, with a $3 \times 3$ block repeated twice

$$
W=\left(\begin{array}{ll}
W_{1} & \\
& W_{1}
\end{array}\right) \quad W_{1}=\left(\begin{array}{lll}
\frac{1}{8 x^{2}} & & \\
& -\frac{1}{16 x^{2}} & \\
& & -\frac{1}{16 x^{2}}
\end{array}\right) .
$$

The proof of 8 shows that the two 2-planes distributions of eigenflag vectors are spanned by $\left\{\partial_{t}, \partial_{x}\right\}$ and $\left\{\partial_{y}, \partial_{z}\right\}$. We deduce from Lemma 19 that our manifold can only be conformal to a product of 2-dimensional manifolds tangent to the planes $\left\langle\partial_{t}, \partial_{x}\right\rangle$ and $\left\langle\partial_{y}, \partial_{z}\right\rangle$.

However, Theorem 15 implies that if our metric were conformal to a product metric, the distribution $\left\langle\partial_{y}, \partial_{z}\right\rangle$ would be umbilical. But in that case, it follows from Definition 13 that the following two numbers should be the same

$$
\begin{aligned}
& g\left(\nabla_{\hat{\partial}_{y}} \hat{\partial}_{y}, \partial_{x}\right)=-\frac{3}{2 x}, \\
& g\left(\nabla_{\hat{\partial}_{z}} \hat{\partial}_{z}, \partial_{x}\right)=\frac{1}{2 x},
\end{aligned}
$$

and we conclude that the metric is not conformal to a product metric.

Thus, the metric in the above Theorem is not covered by our Theorem 9.

It is obvious that $\partial_{t}$ is a parallel vector field, while $\partial_{y}$ and $\partial_{z}$ are unit parallel vector fields for the conformal metrics $1 / x^{3} g$ and $x g$, respectively. Thus, according to Theorem 1 , the coordinate functions $t, y$, and $z$ are LCW's.

In order to check if $\left\langle\partial_{x}\right\rangle$ is a conformal factor, we need to apply our Theorem 15. 
The second fundamental form of $\left\{\partial_{x}\right\}^{\perp}$ in the orthonormal basis $\left\{\partial_{t}, \hat{\partial}_{y}, \hat{\partial}_{z}\right\}$ is:

$$
\left(\begin{array}{ccc}
0 & 0 & 0 \\
0 & -\frac{3}{2 x} & 0 \\
0 & 0 & \frac{1}{2 x}
\end{array}\right)
$$

which is not a multiple of the identity. This means that $\left\{\partial_{x}\right\}^{\perp}$ is not umbilical, and we deduce that $\left\langle\partial_{x}\right\rangle$ is not a conformal factor.

The analysis of the Weyl tensor shows that any possible conformal factor must be contained in either $\left\langle\partial_{t}, \partial_{x}\right\rangle$ or $\left\langle\partial_{y}, \partial_{z}\right\rangle$. Let us push Theorem 15 a little bit further to find all of them.

Let $X$ be a unit vector field in $\left\langle\partial_{t}, \partial_{x}\right\rangle . X$ can be written in the form

$$
X=\cos (\alpha) \partial_{t}+\sin (\alpha) \partial_{x}
$$

for a real valued function $\alpha: M \rightarrow \mathbb{R}$.

If $X$ spans a conformal factor for some $\alpha$, then $\langle X\rangle^{\perp}$ is an umbilical distribution. $X \in\left\langle\partial_{t}, \partial_{x}\right\rangle$ implies $\left\langle\partial_{y}, \partial_{z}\right\rangle \subset\langle X\rangle^{\perp}$, so in particular the form

$$
Z \rightarrow g\left(\nabla_{Z} Z, X\right)
$$

for $Z \in\left\langle\partial_{y}, \partial_{z}\right\rangle$ must be a multiple of the identity. This tensor can be written as a linear combination

$$
g\left(\nabla_{Z} Z, X\right)=\cos (\alpha) g\left(\nabla_{Z} Z, \partial_{t}\right)+\sin (\alpha) g\left(\nabla_{Z} Z, \partial_{x}\right)
$$

but we notice that the first summand is zero

$$
Z \rightarrow g\left(\nabla_{Z} Z, \partial_{t}\right)=0
$$

and we saw in (17) that the matrix of $Z \rightarrow g\left(\nabla_{Z} Z, \partial_{x}\right)$ in the orthonormal basis $\left\{\hat{\partial}_{y}, \hat{\partial}_{z}\right\}$ is not a multiple of the identity. Thus, the only combination of them that produces a multiple of the identity is $\cos (\alpha)=1, \sin (\alpha)=0$.

The same trick will not help us decide whether there are conformal factors of dimension 1 contained in $\left\langle\partial_{y}, \partial_{z}\right\rangle$. Instead, we define

$$
Z=\cos (\alpha) \hat{\partial}_{y}+\sin (\alpha) \hat{\partial}_{z}
$$

and compute the second fundamental form of $Z^{\perp}$ in the basis

$$
\partial_{t}, \quad \partial_{x}, \quad-\sin (\alpha) \hat{\partial}_{y}+\cos (\alpha) \hat{\partial}_{z} .
$$


We remark that for some choices of $\alpha$ the distribution $Z^{\perp}$ is not integrable, which is why the matrix

$$
I I=\left(\begin{array}{ccc}
0 & 0 & 0 \\
0 & 0 & -2 \cos (\alpha) \sin (\alpha) \\
-\partial_{t} \alpha & -\partial_{x} \alpha & \frac{x^{2} \cos (\alpha) \partial_{z} \alpha-\sin (\alpha) \partial_{y} \alpha}{x^{3 / 2}}
\end{array}\right)
$$

is not always symmetric. If $Z^{\perp}$ is umbilical for some choice of $\alpha$, then the above matrix must be a multiple of the identity, and hence it would vanish identically. In particular, $-2 \cos (\alpha) \sin (\alpha)$ is zero, and since $\alpha$ is continuous, we only have two choices: $Z=\hat{\partial}_{y}$ and $Z=\hat{\partial}_{z}$.

REMARK 24. The above example shows how even if the analysis of the Weyl tensor yields an infinite number of candidates to be 1-dimensional conformal factors, a use of Theorem 15 allows to rule out the fake ones. Notice that in this example, it happened that for the false candidates, $Z^{\perp}$ was not umbilical. In the rare event (we do not know of any example) that for false candidates $Z^{\perp}$ was umbilical, the conditions on $H_{1}+H_{2}$ being a gradient field would rule out those eigenflags directions not arising from LCW.

\section{Acknowledgments}

The authors were supported by research grants MTM2011-22612, MTM201128198, MTM2014-57769-1-P and MTM2014-57769-3-P from the Ministerio de Ciencia e Innovación (MCINN), by ICMAT Severo Ochoa projects SEV-20110087 and SEV-2015-0554 (MINECO), and by the ERC 301179.

\section{References}

[1] P. Angulo-Ardoy, 'On the set of metrics without local limiting Carleman weights', Inv. Prob. Imag. 11(1) (2017), 47-64.

[2] P. Angulo-Ardoy, D. Faraco, L. Guijarro and A. Ruiz, 'Obstructions to the existence of limiting Carleman weights', Anal. PDE 9(3) (2016), 575-596.

[3] A. L. Besse, Einstein Manifolds, Ergebnisse der Mathematik und ihrer Grenzgebiete (3) [Results in Mathematics and Related Areas (3)], 10 (Springer, Berlin, 1987).

[4] P. Caro and K. M. Rogers, 'Global uniqueness for the Calderón problem with Lipschitz conductivities', Forum Math. Pi 4 (2016), e2.

[5] P. Caro and M. Salo, 'Stability of the Calderón problem in admissible geometries', Inv. Prob. Imag. 8 (2014), 939-957.

[6] M. P. Do Carmo, Riemannian Geometry Mathematics: Theory and Applications (Birkhaüser Boston, Inc., Boston, MA, 1992). 
[7] D. Dos Santos Ferreira, C. E. Kenig and M. Salo, 'Determining an unbounded potential from Cauchy data in admissible geometries', Comm. Partial Differential Equations 38(1) (2013), 50-68.

[8] D. Dos Santos Ferreira, C. E. Kenig, M. Salo and G. Uhlmann, 'Limiting Carleman weights and anisotropic inverse problems', Invent. Math. 178(1) (2009), 119-171.

[9] D. Dos Santos Ferreira, K. Yaroslav, M. Lassa and M. Salo, 'The Calderon problem in transversally anisotropic geometries', J. Euro. Math. Soc. 18(11) (2016), 2579-2626.

[10] B. Haberman, 'Tataru, Daniel Uniqueness in Calderón's problem with Lipschitz conductivities', Duke Math. J. 162(3) (2013), 496-516.

[11] B. Haberman, 'Uniqueness in Calderón's problem for conductivities with unbounded gradient', Comm. Math. Phys. 340(2) (2015), 639-659.

[12] C. E. Kenig, M. Salo and G. Uhlmann, 'Reconstructions from boundary measurements on admissible manifolds', Inverse Probl. Imaging 5(4) (2011), 859-877.

[13] C. E. Kenig, J. Sjöstrand and G. Uhlmann, 'The Caldern problem with partial data', Ann. of Math. (2) 165(2) (2007), 567-591.

[14] P. D. Lax, Linear Algebra, Pure and Applied Mathematics (Wiley and Sons, New York, 1997).

[15] T. Liimatainen and M. Salo, 'Nowhere conformally homogeneous manifolds and limiting Carleman weights', Inverse Probl. Imaging 6(3) (2012), 523-530.

[16] M. Salo, 'The Calderón problem on Riemannian manifolds', in Inverse Problems and Applications: Inside Out II (ed. G. Uhlmann) (MSRI Publications, Cambridge University Press, Cambridge, 2012).

[17] M. Salo, 'The Calderón problem on Riemannian manifolds', in Inverse Problems and Applications: Inside Out. II, Mathematical Sciences Research Institute Publications, 60 (Cambridge University Press, Cambridge, 2013), 167-247.

[18] J. Sylvester and G. Uhlmann, 'A global uniqueness theorem for an inverse boundary value problem', Ann. of Math. (2) 125 (1987), 153-169.

[19] R. Tojeiro, 'Conformal de Rham decomposition of Riemannian manifolds', Houston J. Math. 32(3) (2006), 725-743. 